is employed then proper ventilation must be insured. The history of mercury poisoning and its manifestation as acrodynia in children is reviewed in an editorial (Clarkson TW. Mercury An element of mystery. N Engl J Med Oct 18, 1990; 323:1137-39). Dimercaptosuccinic acid ${ }^{-} \overline{\text { given }}-\overline{\text { orally }}$ is the most promising therapy.

\title{
CONGENITAL SYPHILIS
}

Seven infants with congenital syphilis who became symptomatic between 3 and 14 weeks of age are reported from the Bronx Municipal Hospital Center and Albert Einstein College of Medicine, Bronx, NY. At delivery four infants and their mothers had negative rapid-plasmareagin tests for syphilis. The other three mothers had been seronegative and were not tested at delivery; two of their infants were seronegative at birth and one was not tested. When the infants became symptomatic all seven and the five mothers available for testing were seropositive for syphilis. A characteristic diffuse rash was the presenting symptom in four and three presented with fever and aseptic meningitis. All infants had multisystem disease evidenced by hepatosplenomegaly, increased aminotransferase and alkaline phosphatase levels, anemia, and monocytosis. All responded to parenteral penicillin. Radiological evidence of bone involvement was seen in three of six patients tested. Renal disease occurred in the youngest child, a three week old boy who had severe nephrosis. A JarischHerxheimer reaction, consisting of a sudden elevation of temperature, occurred in all children within two to six hours after they received the first dose of antibiotics. (Corfman DH, Glaser JH. Congenital syphilis presenting in infants after the newborm period. $\underline{\mathrm{N}}$ Engl $\underline{\mathrm{J}}$ Med Nov 8,$1990 ; 323: 1299-1302$ ).

COMMENT. At least in areas where the disease is prevalent, serologic tests for syphilis should be included in the evaluation of all febrile infants and especially if associated with aseptic meningitis, hepatomegaly, or hematologic abnormalities, even if previous tests for syphilis have been negative at birth. The incidence of syphilis has reached epidemic proportions in some areas. In New York the rate of primary or secondary syphilis in women rose only marginally between 1983 and 1986 but it increased almost four-fold between 1986 and 1988. In the same two year period the number of reported cases of congenital syphilis rose from 57 to 357 . The pediatrician's suspicion of congenital syphilis must remain high to avoid misdiagnosis. (McIntosh $\mathrm{K}$. Congenital syphilis - breaking through the safety net. Edi torial. N Engl J Med Nov 8, 1990; 323:1339-1341).

\section{SEIZURE DISORDERS}

ACETAZOLAMIDE MONOTHERAPY FOR MYOCLONIC EPILEPSY

Chronic acetazolamide monotherapy controlled generalized tonic-clonic seizures in 14 of 31 patients with juvenile myoclonic 
epilepsy treated in the Department of Neurology, Columbia University College of Physicians and Surgeons, New York, NY, and the Department of Neurology, Stamford Hospital, Stamford, CT. The daily dose varied from $500 \mathrm{mg}$ in six patients to $1750 \mathrm{mg}$ in one (average $893 \mathrm{mg}$ ). Acetazolamide was less effective in controlling myoclonus than in the control of generalized tonic-clonic seizures. Six (43\%) of 14 adults with generalized seizures responding to acetazolamide developed renal calculi. (Resor SR, Resor LD. Chronic acetazolamide monotherapy in the treatment of juvenile myoclonic epilepsy. Neurology Nov 1990; $40: 1677-1681$ ).

COMMENT. The frequency of renal calculus as a side effect of chronic acetazolamide therapy in the adults in this study is alarming and sufficient to contraindicate its use. In children, however, renal calculus is a rare side effect of acetazolamide, and this report in adults should not negate the efficacy and clinical use of acetazolamide in the treatment of childhood epilepsy.

In a double blind, placebo controlled trial of acetazolamide in 14 children, ages 6 months to 11 years, an anticonvulsant effect was demonstrated in all patients. Both generalized tonic-clonic and myoclonic seizures were reduced in frequency and in eight patients the maximal reduction in seizures was more than $75 \%$. The control of generalized tonic-clonic seizures was superior to that of the myoclonic type. Acetazolamide monotherapy was used in two patients and additional antiepileptic drugs were continued in the remainder. Tolerance to the effect of acetazolamide shown in eight patients was a greater limiting factor than toxicity in this study. Polyuria and nocturnal enuresis were the only renal side effects and renal calculus did not occur. (Millichap JG. Anticonvulsant action of acetazolamide (Diamox) in children. Neurology $1956 ; 6: 552-559)$. Acetazolamide treatment of absence seizures reviewed in 620 children and young adults provided complete control in $50 \%$ and a $3 / 4$ or greater reduction in an additional $26 \%$. Side effects were reported in 60 (10\%) patients and renal calculus occurred in one, a 20 year old adult. (Millichap JG, Aymat F. Treatment and prognosis of petit mal epilepsy. Ped Clin $\underline{\text { Amer }} 1967 ; \underline{14}: 905-920)$.

MECHANISIS OF ANTIEPILEPTIC DRUG ACTION

The mechanisms of antiepileptic drug action are reviewed from the University Pediatric Epilepsy Program and Division of Pediatric Neurology, University of Minnesota Hospital, Minneapolis, MN. Phenytoin, carbamazepine, and valproic acid decrease sustained repetitive firing of action potentials at therapeutic concentrations. Unlike phenytoin and carbamazepine which block the sodium channel, valproic acid blocks sustained repetitive firing by activation of calcium-dependent, potassium conductance. Phenytoin and carbamazepine also have the ability to block post-tetanic potentiation, an effect mediated by blocking the sodium channel. Benzodiazepines and 